\title{
CORRECTIONS
}

\section{Food fights}

Editor's Choice for the 17 March print issue ("Food fights," BMJ 2018;360:k1190, doi:10.1136/bmj.k1190) mistakenly stated that NICE guidelines "encourage restriction of carbohydrates with a low glycaemic index"; in fact, its guidelines recommend encouraging consumption of carbohydrates with low glycaemic index, such as fruit, vegetables and minimally processed grains, rather than those with high glycaemic index, such as white bread and breakfast cereals. The text has been corrected. 